\title{
Optimum excitation conditions for the generation of high-electric-field terahertz radiation from an oscillator-driven photoconductive device
}

\author{
André Dreyhaupt, Stephan Winnerl, and Manfred Helm \\ Institute of Ion Beam Physics and Materials Research, Forschungszentrum Rossendorf, P.O. Box 510119, \\ 01314 Dresden, Germany \\ Thomas Dekorsy \\ Universität Konstanz, Fachbereich Physik, 78457 Konstanz, Germany
}

\begin{abstract}
Received January 3, 2006; accepted February 15, 2006; posted March 1, 2006 (Doc. ID 66900) We report the impulsive generation of terahertz $(\mathrm{THz})$ radiation with a field amplitude of more than $1.5 \mathrm{kV} / \mathrm{cm}$ at megahertz repetition rates, using an interdigitated photoconducting device. The approach provides an average $\mathrm{THz}$ power of $190 \mu \mathrm{W}$, corresponding to an optical-to- $\mathrm{THz}$ conversion efficiency of 2.5 $\times 10^{-4}$. Optimum conditions are achieved when the excitation spot size is of the order of the $\mathrm{THz}$ wavelength.
\end{abstract}

Impulsive terahertz $(\mathrm{THz})$ sources have undergone significant improvements over the past decade. For example, field amplitudes up to $400 \mathrm{kV} / \mathrm{cm}$ have been demonstrated by four-wave mixing in ionized air by using amplified laser systems. ${ }^{1}$ For many applications oscillator-based $\mathrm{THz}$ systems are preferable, since they are less complex and allow high signal-to-noise ratios to be achieved. To this end, several new approaches to photoconductor (PC)-based $\mathrm{THz}$ emitters excited by high-repetition-rate femtosecond oscillators have been presented. ${ }^{2,3} \mathrm{THz}$ applications range from novel materials ${ }^{4}$ and spectroscopy ${ }^{5}$ to imaging. ${ }^{6}$ In this Letter we report a PC THz emitter operated at moderate voltages and a megahertz repetition rate, providing high $\mathrm{THz}$ electric fields and power.

Our concept is based on an interdigitated gold finger electrode structure with $150 \mathrm{~nm}$ thickness and $5 \mu \mathrm{m}$ electrode spacing fabricated on a semiinsulating GaAs substrate followed by a $\mathrm{Si}_{3} \mathrm{~N}_{4}$ insulation layer of $1 \mu \mathrm{m}$ thickness. A further metallization covers every second finger electrode spacing. The active area of this structure is $3 \mathrm{~mm} \times 3 \mathrm{~mm}$, and it provides a $\mathrm{THz}$ source that is operated by a femtosecond near-infrared (NIR) laser system (800 $\mathrm{nm}$ central wavelength, $65 \mathrm{fs}$ full width at half-maximum (FWHM) pulse duration, $78 \mathrm{MHz}$ repetition rate, maximum $800 \mathrm{~mW}$ average power). Hence a broadband single-cycle electromagnetic wave is emitted by the photoexcited carriers, accelerated in the electric field between the electrodes. Owing to the opaque second gold metallization on top of the finger electrodes, photoexcitation takes place only in uniform field regions. The resulting $\mathrm{THz}$ radiation interferes constructively in the far field. This device overcomes disadvantages inherent to emitters using photoconductive antennas (usually low power) and largeaperture emitters using two stripe electrodes (limited bandwidth, high voltages required). A detailed de- scription of the emitter concept was given in an earlier paper.

Two off-axis parabolic mirrors (50 and $25 \mathrm{~mm}$ effective focal length) are used to focus the $\mathrm{THz}$ radiation to the detection system as shown in the inset of Fig. 1(a). A small fraction of the laser beam is split off for the $\mathrm{THz}$ detection. This optical beam is realigned with the $\mathrm{THz}$ beam by a tin-doped indium oxide (ITO) coated mirror. ${ }^{8}$ The $\mathrm{THz}$ detection is performed by conventional electro-optic sampling ${ }^{9,10}$ (EOS), using a $25 \mu \mathrm{m} \mathrm{ZnTe} \mathrm{(110)} \mathrm{crystal} \mathrm{on} \mathrm{fused} \mathrm{silica,} \mathrm{a} \mathrm{polarizing}$ beam splitter, two balanced photodiodes, and a lock-in amplifier locked to the $10 \mathrm{kHz}$ modulation of the $\mathrm{THz}$ emitter bias. The duty cycle of the bias was $10 \%$ to limit the power dissipation that may reduce the THz output. The setup was purged with nitrogen to reduce the absorption of $\mathrm{THz}$ radiation by atmospheric water vapor.

Figure 1(a) shows the $\mathrm{THz}$ electric field at different acceleration fields. For a bias of $30 \mathrm{~V}(60 \mathrm{kV} / \mathrm{cm})$ and $4 \times 10^{17} / \mathrm{cm}^{3}$ excitation density within a $300 \mu \mathrm{m}$ (FWHM) NIR spot, a THz electric field of $1.7 \mathrm{kV} / \mathrm{cm}$ was deduced by using the method described in Ref. 2 . Until now this is the largest $\mathrm{THz}$ field reported in the literature (to our knowledge) for an oscillator-excited $\mathrm{THz}$ source operated at megahertz repetition rates. The strong increase of the detected $\mathrm{THz}$ field compared with that of our earlier paper ${ }^{7}(85 \mathrm{~V} / \mathrm{cm}$ at $100 \mathrm{~mW}$ excitation power) is based on the optimization of the excitation conditions (see discussion below). However, a maximum signal-to-noise ratio is achieved by using a thicker ZnTe analyzer and a 50\% duty cycle. That is to say, with a $1 \mathrm{~mm} Z \mathrm{ZnTe}$ crystal and $50 \%$ duty cycle the signal-to-noise ratio is about $3 \times 10^{4}$, measured within $25 \mathrm{~s}$. The measurement time is limited by the slow optical delay in step-scan mode. This corresponds to a $2 \%$ differential signal at the photodiodes. The resulting spectra in Fig. 1(b) show broadband frequency characteristics with a 

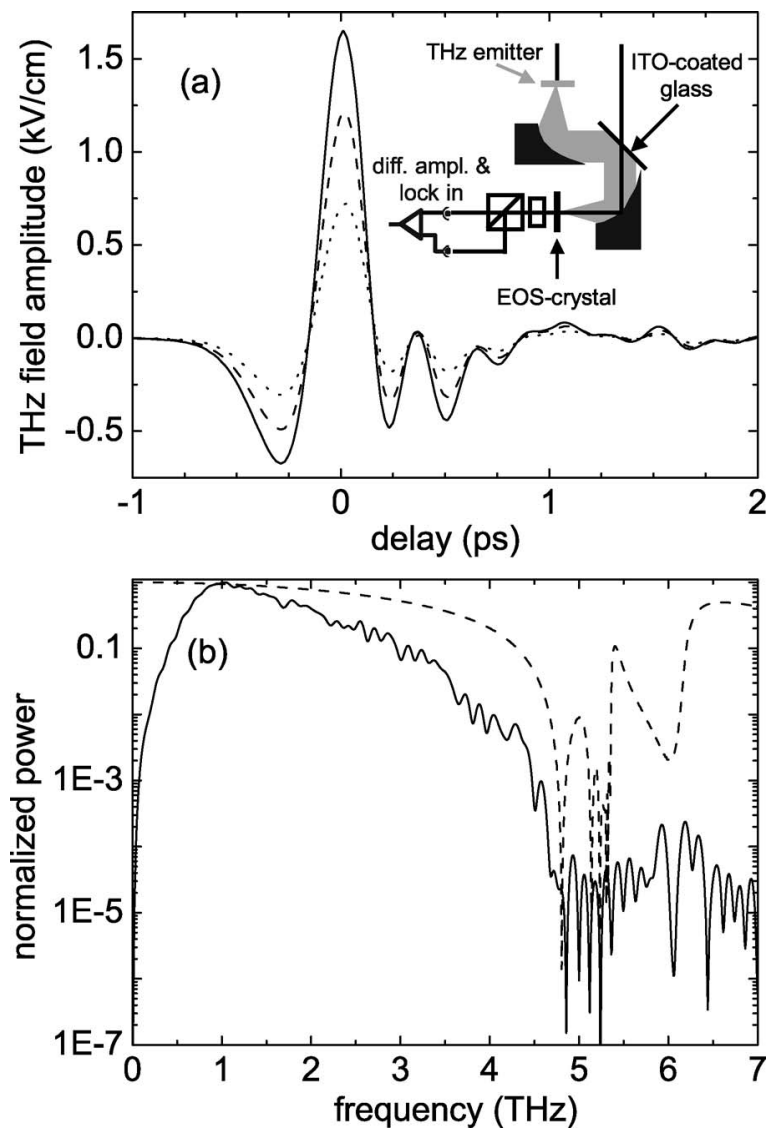

Fig. 1. (a) Time-domain $\mathrm{THz}$ waveform for acceleration fields of $20 \mathrm{kV} / \mathrm{cm}$ (dotted), $40 \mathrm{kV} / \mathrm{cm}$ (dashed), and $60 \mathrm{kV} / \mathrm{cm}$ (solid) at an excitation density of $4 \times 10^{17} \mathrm{~cm}^{-3}$. (b) Power spectra calculated from the time-domain data of (a) for $60 \mathrm{kV} / \mathrm{cm}$. The dashed curve represents the calculated response of the $\mathrm{ZnTe}$ analyzer crystal.

$10 \mathrm{~dB}$ bandwidth of $2.5 \mathrm{THz}$. Apart from that the spectra are influenced by phonons of the ZnTe crystal, the mismatch of the phase and group velocity of the $\mathrm{THz}$ and NIR beams, respectively and residual water vapor in the setup.

The calculation of the response of the ZnTe EOS crystal takes into account the velocity mismatch of the $\mathrm{THz}$ wave and the $800 \mathrm{~nm}$ radiation and the frequency dependence of the electro-optic coefficient $r_{41}$ and of the reflections of the $\mathrm{THz}$ beam. ${ }^{10}$ The pulse duration of the probe beam ( $65 \mathrm{fs}$ ) is included. Material constants in the calculation are taken from Ref. 10 and references therein, except for the highfrequency dielectric constant of $\mathrm{ZnTe}$, where $\epsilon_{\infty}=7.3$ is used ${ }^{11}$ to fit our experimental data. The group velocity dispersion of $\mathrm{ZnTe}$ in the wavelength region around $800 \mathrm{~nm}$ is modeled by a quadratic fit to experimental data for the refractive index of Ref. 11. These data are in good agreement with more recent experimental studies. ${ }^{12,13}$ We tested our model by comparing the calculated response with experimental spectra for different ZnTe thicknesses (not shown) and found very good agreement. As indicated in Fig. 1(b), the emitted $\mathrm{THz}$ radiation extends up to 4.5 $\mathrm{THz}$.

The spatial distribution of the $\mathrm{THz}$ spot at the electro-optic sensor was mapped by steering the $\mathrm{THz}$ beam with the ITO mirror while keeping the NIR probe beam unchanged. The $\mathrm{THz}$ beam has an elliptical Gaussian shape as displayed in the inset of Fig. 2 , with sizes of 340 and $410 \mu \mathrm{m}$ FWHM. From the knowledge of the temporal evolution, spatial distribution, and maximum electric field of the $\mathrm{THz}$ pulse, we have calculated the average $\mathrm{THz}$ power within the bias pulse to $190 \mu \mathrm{W}$ for an optical excitation power of $775 \mathrm{~mW}$ and an acceleration field of $60 \mathrm{kV} / \mathrm{cm}$. This corresponds to a NIR-to-THz powerconversion efficiency of about $2.5 \times 10^{-4}$. It should be noted that in the present device less than $20 \%$ of the incident radiation is absorbed in the PC due to $75 \%$ coverage of the surface with metallization and the reflectivity of the PC. This value can be enhanced by using an asymmetric finger structure minimizing the shadowed region between the electrodes and by adapting the dielectric cover layers to an antireflection coating. Only recently a power-conversion efficiency of about $7 \times 10^{-4}$ was published by Kim et al. ${ }^{3}$ for line-shape excitation near one electrode of a coplanar antenna. However, the emitted $\mathrm{THz}$ power was only of the order of $10 \mu \mathrm{W}$.

We have also measured the emitted $\mathrm{THz}$ power directly by using a pyroelectric detector (Fig. 2) and found a quadratic dependence on the optical excitation power. For an excitation power of $800 \mathrm{~mW}$ and an acceleration field of $40 \mathrm{kV} / \mathrm{cm}$ we have achieved an average $\mathrm{THz}$ power of $24 \mu \mathrm{W}$. Taking into account the different parameters (electric field and duty cycle), the power measured with the pyroelectric detector was about 3.5 times smaller than the value obtained from the EOS measurement, which is probably due to an insufficient free aperture of the detector and an uncertainty in the calibration.

To find the optimal operating conditions, we have investigated the dependence of the $\mathrm{THz}$ field on the radius $a$ of the optically excited area. The $\mathrm{THz}$ emission of our source can be treated as transmission through a hole in the limiting cases $a \ll \lambda$ and $a \gg \lambda$. For $a \gg \lambda$ all radiated frequency components can propagate to the far field. Assuming a circular excitation spot and an invariant $\mathrm{THz}$ beam profile at the

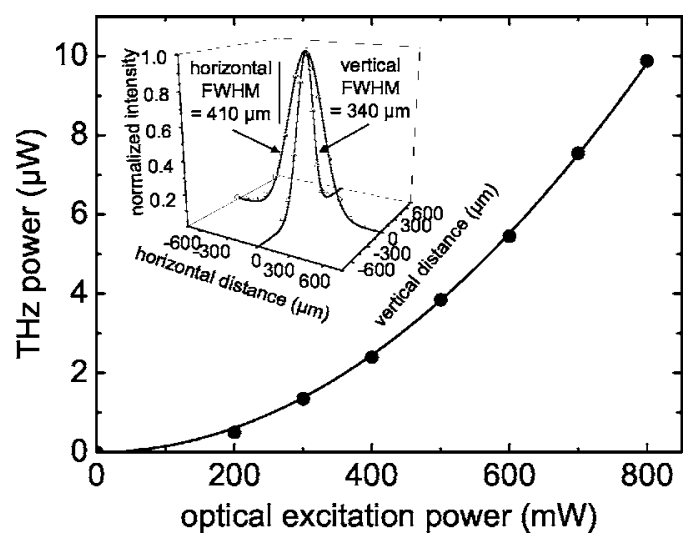

Fig. 2. Dependence of the $\mathrm{THz}$ power (measured with a pyroelectric detector) on excitation power using an acceleration field of $20 \mathrm{kV} / \mathrm{cm}$. Filled circles, measured data; solid curve, second-order polynomial. Inset, beam profile of the $\mathrm{THz}$ spot at the electro-optic sensor. 


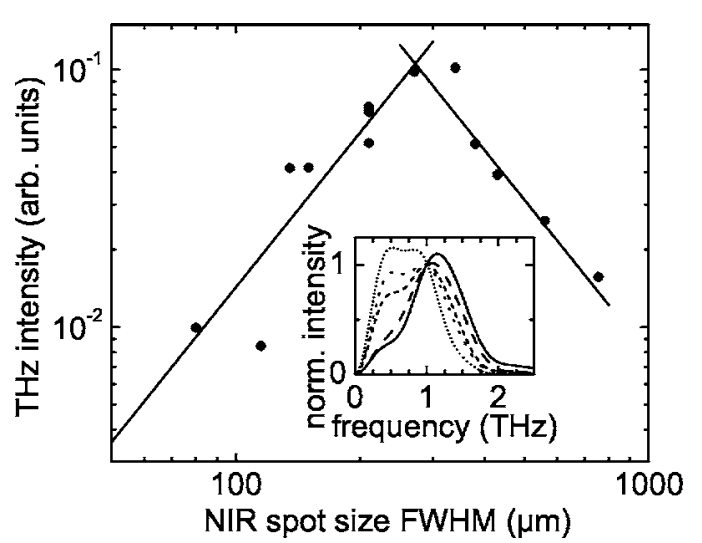

Fig. 3. Dependence of the detected $\mathrm{THz}$ intensity $\left(E_{\mathrm{THz}}^{2}\right)$ on the size (FWHM) of the exciting NIR spot (double logarithmic scale). Filled circles, measured data; solid lines, guides to the eyes. Inset, $\mathrm{THz}$ spectra for various excitation spot sizes normalized to the intensity value at $1 \mathrm{THz}$; $755 \mu \mathrm{m}$ (short dotted), $560 \mu \mathrm{m}$ (dotted), $430 \mu \mathrm{m}$ (short dashed), $210 \mu \mathrm{m}$ (dashed), $115 \mu \mathrm{m}$ (solid).

electro-optic crystal, the NIR power $P_{\mathrm{NIR}}$ is related to the $\mathrm{THz}$ intensity $I_{\mathrm{THz}}$ by

$$
\begin{gathered}
P_{\mathrm{NIR}} / \pi a^{2}=I_{\mathrm{NIR}}, \\
I_{\mathrm{NIR}} \propto n \propto E_{\mathrm{THz}}, \quad E_{\mathrm{THz}}^{2} \propto I_{\mathrm{THz}},
\end{gathered}
$$

where $I_{\mathrm{NIR}}$ is the optical intensity, $n$ is the carrier density, and $E_{\mathrm{THz}}$ is the $\mathrm{THz}$ electric field. Assuming that the $\mathrm{THz}$ radiating area equals half the optically excited area, the $\mathrm{THz}$ power $P_{\mathrm{THz}}$ is given by

$$
P_{\mathrm{THz}}=\frac{\pi a^{2}}{2} I_{\mathrm{THz}}
$$

and with Eq. (1),

$$
P_{\mathrm{THz}} \propto P_{\mathrm{NIR}}^{2} / a^{2} .
$$

Thus for a given optical power the $\mathrm{THz}$ power decreases with $1 / a^{2}$. If $a \ll \lambda$ the wave propagation is diffraction limited. This is reflected in the spectra shown in the inset of Fig. 3, which exhibit a blueshift for smaller excitation spots, because only short wavelengths propagate to the far field. The $\mathrm{THz}$ power in the far field is proportional to $a^{2}$, since an additional factor as derived by Bethe ${ }^{14}$ and recently discussed by García de Abajo ${ }^{15}$ with an $a^{4}$ dependence enters Eq. (2).

Therefore the maximum power-conversion efficiency is achieved for an excitation spot size of the order of the central $\mathrm{THz}$ wavelength $(\approx 300 \mu \mathrm{m}$ in our experiments) as long as the emitter is not saturated owing to screening. However, amplifier-driven systems may use larger excitation spot sizes to prevent saturation ${ }^{16}$ and achieve higher output with a similar conversion efficiency if $I_{\text {NIR }}$ is kept constant. We have measured the $\mathrm{THz}$ intensity for different optical spot sizes in between the above limiting cases; thus the above discussion does not strictly apply. However, Fig. 3 exhibits a maximum at about $300 \mu \mathrm{m}$ and a de- crease of the $\mathrm{THz}$ intensity for smaller and larger spot sizes. A similar behavior was reported for optical rectification experiments by Xu and Zhang. ${ }^{17}$ In contrast to our investigations they measured the $\mathrm{THz}$ power (as opposed to the intensity).

Large-aperture $\mathrm{THz}$ emitters typically exhibit $\mathrm{THz}$ spectra ${ }^{16}$ centered at relatively low frequencies. This is usually attributed to the low acceleration field within the large distance between the electrodes. However, those emitters are usually excited by a large NIR spot, which as well enhances the fraction emitted at low frequencies $(<1 \mathrm{THz})$. On the other hand, the excellent properties of the semilargeaperture GaAs emitter proposed by Planken et al. ${ }^{2}$ can be ascribed to the fact that the excitation spot size is of the order of the main $\mathrm{THz}$ emission wavelength.

In summary we have presented a bright source of $\mathrm{THz}$ radiation with a kilovolt per centimeter $\mathrm{THz}$ field amplitude and high power efficiency excited by an oscillator at a megahertz repetition rate. It has been demonstrated that the optimum excitation spot size is of the order of the central wavelength of the $\mathrm{THz}$ wave.

We acknowledge valuable discussion with $\mathrm{C}$. Kübler and R. Huber concerning the preparation of thin ZnTe crystals. S. Winnerl's e-mail address is s.winnerl@fz-rossendorf.de; A. Dreyhaupt's e-mail address is a.dreyhaupt@fz-rossendorf.de.

\section{References}

1. T. Bartel, P. Gaal, K. Reimann, M. Woerner, and T. Elsaesser, Opt. Lett. 30, 2805 (2005).

2. P. C. M. Planken, C. E. W. M. van Rijmenam, and R. N. Schouten, Semicond. Sci. Technol. 20, S121 (2005), and references therein.

3. J. H. Kim, A. Polley, and S. E. Ralph, Opt. Lett. 30, 2490 (2005).

4. H. O. Moser, B. D. F. Casse, O. Wilhelmi, and B. T. Saw, Phys. Rev. Lett. 94, 063901 (2005).

5. N. Sekine and K. Hirakawa, Phys. Rev. Lett. 94, 057408 (2005).

6. M. Usami, M. Yamashita, K. Fukushima, C. Otani, and K. Kawase, Opt. Lett. 86, 141109 (2005).

7. A. Dreyhaupt, S. Winnerl, T. Dekorsy, and M. Helm, Appl. Phys. Lett. 86, 121114 (2005).

8. T. Bauer, J. S. Kolb, T. Löffler, E. Mohler, H. G. Roskos, and U. C. Pernisz, J. Appl. Phys. 92, 2210 (2002).

9. Q. Wu and X.-C. Zhang, Appl. Phys. Lett. 68, 1604 (1996).

10. A. Leitenstorfer, S. Hunsche, J. Shah, M. C. Nuss, and W. H. Knox, Appl. Phys. Lett. 74, 1516 (1999).

11. T. R. Sliker and J. M. Jost, J. Opt. Soc. Am. 56, 130 (1966).

12. K. Sato and S. Adachi, J. Appl. Phys. 73, 926 (1993).

13. Q. Wu and X.-C. Zhang, IEEE J. Solid-State Circuits 2, 693 (1996).

14. H. A. Bethe, Phys. Rev. 66, 163 (1944).

15. F. J. García de Abajo, Opt. Express 10, 1475 (2002).

16. T. Löffler, M. Kreß, M. Thomson, T. Hahn, N. Hasegawa, and H. G. Roskos, Semicond. Sci. Technol. 20, S134 (2005).

17. J. Z. Xu and X.-C. Zhang, Opt. Lett. 27, 1067 (2002). 